\title{
sciendo
}

\section{IS CONTAMINATION OF BOVINE-SOURCED MATERIAL WITH BOVINE VIRAL DIARRHEA VIRUS STILL A PROBLEM IN COUNTRIES WITH ONGOING ERADICATION CAMPAIGNS?*}

\author{
Aleksandra Antos ${ }^{1}$, Jerzy Rola ${ }^{1}$, Michał Bednarski ${ }^{2}$, Michał Konrad Krzysiak ${ }^{3,4}$, \\ Julia Kęsik-Maliszewska ${ }^{1}$, Magdalena Larska ${ }^{1 *}$ \\ ${ }^{1}$ Department of Virology, National Veterinary Research Institute, Al. Partyzantów 57, \\ 24-100 Puławy, Poland \\ ${ }^{2}$ Department of Epizootiology and Clinic of Bird and Exotic Animals, \\ Wrocław University of Environmental and Life Sciences, Pl. Grunwaldzki 45, 50-366 Wrocław, Poland \\ ${ }^{3}$ Białowieża National Park, Park Pałacowy 11, 17-230 Białowieża, Poland \\ ${ }^{4}$ Institute of Forest Sciences, Faculty of Civil Engineering and Environmental Sciences, Białystok \\ University of Technology, Wiejska 45 E, 15-351 Białystok, Poland \\ •Corresponding author: m.larska@piwet.pulawy.pl
}

\begin{abstract}
In this report, we describe the detection of bovine viral diarrhea virus (BVDV) contamination in commercial animal-derived sera and vaccines against animal viral pathogens on the market in Poland. Antibodies against BVDV were detected in $4 / 45$ sera samples $(8.9 \%)$ using an ELISA test. The presence of BVDV antigen was found using ELISA in 3/45 serum samples $(6.6 \%)$ and $18 / 172$ vaccine samples $(10.5 \%)$. An RT-PCR was conducted using primers targeting two genome regions, the five prime untranslated region (5'UTR) and $\mathrm{N}$-terminal protease $\left(\mathrm{N}^{\text {pro }}\right)$. BVDV RNA was detected in $33 / 45(73.3 \%)$ of sera, and $11 / 172$ samples $(6.4 \%)$ of collected vaccines, of which one vaccine did not declare BVDV strain in its composition. A single serum showed the presence of an infectious virus and only one was contaminated with all 3 species of BVDV. The most frequent species in sera was BVDV-3 (75.5\%), whereas in vaccines only BVDV-1 was identified. Sequence analysis showed that the tested commercial sera and one vaccine were contaminated by six genotypes of BVDV: $-1 \mathrm{a},-1 \mathrm{~b},-1 \mathrm{c},-1 \mathrm{~d},-\mathbf{2}$, and -3 . Identification of BVDV and its genetic material in animal-derived products is important due to the possibility of pestivirus transmission as well as the chance of falsifying the results of a diagnostic test. It also demonstrates the necessity of rigorous monitoring of the bioproducts used at the laboratory and industry level.
\end{abstract}

Key words: fetal bovine serum, vaccine, pestivirus, viral contamination

Beside bacterial, fungal, and mycoplasma contamination, viral contamination is an important safety risk for all bioproducts manufactured using cell culture. Never-

\footnotetext{
*This work was supported by the National Science Centre, Poland (grant number 2012/07/E/ NZ6/01726).
} 
theless, the contamination by viruses is very problematic to prevent, control, detect, and efficiently remove from stock materials used in the manufacturing process. The fetal bovine serum (FBS) broadly used in the production of vaccines and biotherapeutics may be contaminated with a broad spectrum of viruses. Among them, due to their worldwide prevalence in the cattle population, pestiviruses such as the bovine viral diarrhea virus (BVDV), play a major role as contaminants of bovine-sourced materials. BVDV is considered one of the most important infectious diseases of cattle with respect to animal health and economic impact worldwide. Epidemiological data from Poland indicated that infection with BVDV is very common, with over $70 \%$ of dairy herds seropositive in bulk-tank milk testing (Kuta et al., 2013 a). A similar study carried out by Rypuła et al. (2010) showed a high percentage of BVDV-positive animals, especially in large dairy herds. The presence of the BVDV in beef cattle aged less than 12 months in Poland was also reported by Wernicki et al. (2015). Furthermore, the constantly growing number of genetic variants recently identified in Poland shows that this problem is still relevant (Mirosław and Polak, 2019). Some European countries, including Denmark, Norway, Sweden, and Finland have recently eradicated or are almost free from BVDV. Switzerland, Austria, and Germany are in the advanced or final stages of BVDV eradication, whereas the Netherlands and Ireland have only recently implemented control programs (Wernike et al., 2017). Following these countries, Poland introduced a voluntary BVDV eradication program in early 2018 . For the eradication program to be successful, it is crucial to control the risk factors for possible virus transmission including contamination in animal-derived products such as vaccines, which can introduce an infectious virus to a herd.

BVDV species 1 (Pestivirus A) and 2 (Pestivirus B), each comprising several subgenotypes, together with Classical Swine Fever Virus (CSFV, Pestivirus C), and Border Disease Virus (BDV, Pestivirus D) belong to the genus Pestivirus of the family Flaviviridae (Smith et al., 2017). BVDV-1 is the most common species of this virus around the world, as well as the most prevalent in Poland (Kuta et al., 2013 b; Mirosław and Polak, 2019), whereas BVDV-2 is common in North and South America. However, the transmission of species 2 to European herds was described (Vilcek et al., 2005; Polak et al., 2014). Additionally, a novel putative pestivirus species was discovered in the strain named D32/00_'HoBi' in contaminated commercial FBS of Brazilian origin (Schirrmeier et al., 2004). It was followed by the detection of natural infection with this pathogen in southeast Asia, Italy, and Brazil (Stahl et al., 2007; Decaro et al., 2011; Cortez et al., 2006). This new species is tentatively called "HoBi-like", "BVDV-3" or "atypical pestivirus (Pestivirus H)" (Smith et al., 2017). All pestiviruses are structurally and genetically related and serological cross-reactivity between members of the genus has been reported (Bauermann et al., 2012). Based on the induced effect on cell culture, two biotypes of BVDV were distinguished: the noncytopathic (ncp) and cytopathic (cp). The key issue of the BVDV infection (uniquely ncp BVDV infection) is its ability to generate persistent infection through fetal infection in early gestation, these fetuses then serving as virus reservoirs. Infected fetuses are the source of high loads of virus, and therefore contaminate any subsequently produced FBS (Deregt and Loewen, 1995). BVDV almost always spreads by 
direct contact with persistently infected (PI) animals through introduction or mixing at a show, sale, feedlot or through a fence. Other sources of infection besides the PI animals include transiently infected (TI) animals, wild ruminants and nonbovine hosts, and BVDV-contaminated semen and embryos. Additionally, the spread of the virus via needles, nose tongs, hoof trimmers, and vaccine vials was also proved.

FBS is the most commonly used supplement in cell culture media since it contains high levels of nutrients, adhesion proteins, and growth factors (GF), among other vital components. Cell cultures have wide clinical application in medicine, such as in the production of vaccines, diagnostic tests, and cell therapy (Hawkes, 2015). Previously conducted research has shown that pestivirus contamination is common in FBS (Audet et al., 2000; Polak et al., 2008; Laassri et al., 2018; Pecora et al., 2019) and can also be present in bovine-sourced products for an animal or human use (Barkema et al., 2001; Giangaspero et al., 2001; Studer et al., 2002). The presence of BVDV was confirmed for instance in the measles, mumps, rubella, and polio vaccines administered to children (Giangaspero et al., 2001; Sasaki et al., 1996). Although pestiviruses are not considered zoonotic, their antigens were found in the feces of children showing signs of gastroenteritis and respiratory disorders (Yolken et al., 1989). Furthermore, BVDV was isolated from human leukocytes in patients in Belgium (Giangaspero et al., 1997). Recent findings also confirmed the presence of peptide(s) from the polyprotein of a bovine-like viral diarrhea virus in Zika-positive brains of microcephalic fetuses during the 2015 outbreak in Brazil (Nogueira et al., 2016). In addition to exposing of animals and humans to BVDV, the contamination can affect in vitro biological and diagnostic tests, including those to diagnose infection with related viruses such as classical swine fever virus (CSFV) and human hepatitis $\mathrm{C}$ virus (HCV).

At present, with the common use of techniques such as heat inactivation, ultrafiltration, ethylene-imine inactivation, or gamma irradiation, the risk of transmission of pathogens has nearly been eliminated, but does still exist. The aim of the conducted research was to determine the potential contamination of commercial FBS and vaccines against animal viral and bacterial pathogens which are manufactured containing animal-derived products. The conducted research will allow estimation of how widely pestiviruses are unintentionally distributed in products presumed to be safe, and assessment of the risk of infection by using such products available on the Polish market. The safety of animal-derived products is a crucial issue in avoiding the risk of pathogen introduction into nonendemic regions or unintentional induction of an immune response in herds where an eradication campaign pursues a strategy excluding vaccination.

\section{Material and methods}

\section{Samples}

We tested 45 commercial sera including fetal bovine/calf serum - FBS/FCS $(n=33)$, newborn bovine/calf serum - NBS/NCS $(n=2)$, fetal equine serum - FES $(n=2)$, and horse serum - HS $(n=8)$ produced in the years 1990-2016 and obtained 
from 13 commercial suppliers. We also assayed 172 vaccines against viral $(n=121)$, bacterial $(n=31)$, and both viral and bacterial $(n=20)$ disease for different animal species like swine, cows, horses, sheep, goats, ducks and geese, chickens, and rabbits. The tested vaccines were different types: attenuated, inactivated, live, live attenuated, live recombinant, modified live, toxoids, subunit, and protein. All commercial products were purchased from Polish market suppliers and were tested for the presence of antibodies against BVDV, BVDV antigen, and its genetic material.

\section{BVDV antibody detection}

The presence of BVDV antibodies in sera was tested with a commercial enzymelinked immunosorbent assay (ELISA) kit. An Ab ELISA based on the pestivirus envelope protein $\mathrm{E}^{\mathrm{rns}}$ (BVDV Total Ab Test, IDEXX, Liebefeld-Bern, Switzerland) was used according to the manufacturer's instructions. The test provides specificity and sensitivity of respectively $97.1 \%$ and $96.7 \%$ compared with the virus neutralization test (VNT) (Lanyon et al., 2013).

\section{BVDV antigen detection}

A commercial ELISA which detects all three species of BVDV, based on the BVDV E $E^{\text {rns }}$ antigen (BVDV Ag/Serum Plus, IDEXX, Liebefeld-Bern, Switzerland) was used. Tests were carried out on sera and vaccine samples according to the manufacturer's protocol. This test demonstrates specificity of more than $99.7 \%$ and a sensitivity of nearly 100\% (Wolf, 2007; Van Maanen, 2001; Idexx Laboratories, 2005).

\section{Total RNA extraction and BVDV genome detection}

Extraction of viral nucleic acid from sera, vaccines, and potentially infected cell culture was performed with a QIAamp Viral RNA Mini Kit (Qiagen, Hilden, Germany) according to the manufacturer's spin protocol. Extracted RNA samples were stored at $-80^{\circ} \mathrm{C}$ prior to PCR tests. The samples were tested individually.

RT-PCR

A standard one-step RT-PCR was performed using a Transcriptor One-Step RTPCR Kit (Roche, Mannheim, Germany) using the provided primers and adhering to the manufacturer's instructions (Table 1). The final volume of RT-PCR mixture was $25 \mu \mathrm{l}$, comprising: $15.5 \mu \mathrm{l}$ of RNase free water, $5 \mu \mathrm{l}$ of reaction buffer, $1 \mu \mathrm{l}$ of each primer $(10 \mu \mathrm{M}), 0.5 \mu \mathrm{l}$ of enzyme mix, and $2 \mu \mathrm{l}$ of RNA template. The reaction conditions were set at $50^{\circ} \mathrm{C}$ for $30 \mathrm{~min}$ and $94^{\circ} \mathrm{C}$ for $7 \mathrm{~min}$, followed by 10 cycles of $94^{\circ} \mathrm{C}$ for $10 \mathrm{~s}, 53^{\circ} \mathrm{C}$ for $30 \mathrm{~s}, 68^{\circ} \mathrm{C}$ for $30 \mathrm{~s}$, then followed by 25 cycles of $94^{\circ} \mathrm{C}$ for $10 \mathrm{~s}, 53^{\circ} \mathrm{C}$ for $30 \mathrm{~s}$, and $68^{\circ} \mathrm{C}$ for $33 \mathrm{~s}$ with a final extension step of $68^{\circ} \mathrm{C}$ for $7 \mathrm{~min}$. RT-PCR products were analyzed with a molecular weight standard on $1.5 \%$ agarose gel stained with ethidium bromide.

\section{Real-time RT-PCR}

An in-house real-time RT-PCR which allows species differentiation was performed using AgPath-ID Kit reagents (Life Technologies, Waltham, MA, USA) according to the manufacturer's protocol in the StepOnePlus real-time PCR system (Life Technologies, Republic of Singapore). Details of the primers and probes are 
shown in Table 1. The assay was run using the following thermal program: $48^{\circ} \mathrm{C}$ for $10 \mathrm{~min}$ (RT-step), $95^{\circ} \mathrm{C}$ for $10 \mathrm{~min}$, and 40 cycles of $95^{\circ} \mathrm{C}$ for $15 \mathrm{~s}$ and $60^{\circ} \mathrm{C}$ for $45 \mathrm{~s}$. The reaction was performed for 42 cycles with a $\mathrm{Ct}$ (threshold cycle) value of 40 as a cut-off.

Table 1. Primers and probes used in this study

\begin{tabular}{|c|c|c|c|c|}
\hline & $\begin{array}{c}\text { Primers } \\
\text { and probes }\end{array}$ & Sequence $\left(5^{\prime} \rightarrow 3^{\prime}\right)$ & Target & Reference \\
\hline \multirow{8}{*}{ 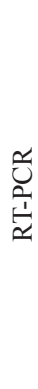 } & $324 \mathrm{~F}$ & ATG CCC WTA GTA GGA CTA GCA & \multirow{2}{*}{ BVDV-1,2,3/5'UTR } & \multirow[t]{2}{*}{ Vilcek et al., 1994} \\
\hline & $326 \mathrm{R}$ & TCA ACT CCA TGT GCC ATG TAC & & \\
\hline & $\mathrm{B} 32 \mathrm{~F}$ & TGCTACTAAAAATCTCTGCTGT & \multirow{2}{*}{ BVDV-1/Npro } & \multirow[t]{2}{*}{ Toplak et al., 2004} \\
\hline & $\mathrm{B} 32 \mathrm{R}$ & CCATCTATrCAyACATArATGTGGT & & \\
\hline & Npro_LVF & CTGTACATGGCACATGGAGTTG & \multirow[b]{2}{*}{ BVDV-2/N $\mathrm{N}^{\text {pro }}$} & \multirow{2}{*}{$\begin{array}{l}\text { Weber et al., } 2014 \\
\text { (with } \\
\text { modification) }\end{array}$} \\
\hline & Npro_LVR & AARCTGTTGTATACCCATTT & & \\
\hline & SF1 & GACTAGTGGTGGCAGTGAGC & \multirow{2}{*}{ BVDV-3/Npro } & \multirow[t]{2}{*}{ Liu et al., 2009} \\
\hline & SR1 & GCAGCTTCCTACCCAGATGG & & \\
\hline \multirow{7}{*}{ 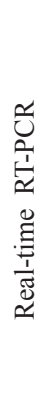 } & Pesti-F & CTAGCCATGCCCTTAGTAG & \multirow{2}{*}{ BVDV-1,2 } & \multirow[t]{2}{*}{ Baxi et al., 2006} \\
\hline & Pesti-R & CGTCGAACCAGTGACGACT & & \\
\hline & BVDV-1-Pr & $\begin{array}{c}\text { FAM-TAGCAACAGTGGT- } \\
\text { GAGTTCGTTGGATGGCT-BHQ1 }\end{array}$ & BVDV-1 & \multirow{5}{*}{ Liu et al., 2008} \\
\hline & BVDV-2-Pr & $\begin{array}{c}\text { VIC-TAGCGGTAGCAGT- } \\
\text { GAGTTCGTTGGATGGCC-BHQ1 }\end{array}$ & BVDV-2 & \\
\hline & T134-F & GACTAGTGGTGGCAGTGAGC & \multirow{3}{*}{ BVDV-3 } & \\
\hline & T220-R & GAGGCATTCCTTGATGCGTC & & \\
\hline & T155r-P & $\begin{array}{c}\text { FAM-ACTCGGGGCTTCGGTGATC- } \\
\text { CAGGG-BHQ1 }\end{array}$ & & \\
\hline
\end{tabular}

\section{Assessment of BVDV infectious status in commercial sera}

To determine if the virus could be infectious for living organisms, all BVDV antigens and genome-positive sera were used to prepare a growing medium for the propagation of Madin-Darby bovine kidney (MDBK) cell lines. Before the experiment, the medium and cell culture were checked for BVDV presence. Heat inactivation of sera was not carried out before testing in order to get reliable information about the effectiveness of virus reduction/removal by the manufacturer. MDBK cells were split twice with the addition of tested BVDV-positive sera. Before the third splitting, the cells were washed with pure EMEM medium and one growing medium with contaminated serum was excluded from further incubation. After 3 days, cells were harvested and frozen until analysis. RNA was extracted from cell culture samples, and an RT-PCR with the panpestivirus primer set and a real-time RT-PCR were conducted (details described in section 2.4.1). 


\section{Sequencing and phylogenetic analysis}

The products of the standard RT-PCR with all primers listed in Table 1 were purified and sequenced as described previously (Kuta et al., 2013 b). Phylogenetic analysis was done by the neighbor-joining (NJ) statistical method with the Kimura two-parameter model using MEGA software (version 5.03). The reliability of the constructed phylogenetic trees was evaluated by running 1000 bootstrap replications in the phylogeny test and bootstrap values greater than or equal to $70 \%$ were considered good support.

\section{Results}

\section{BVDV antibodies detection}

One sample of FBS and two of NBS/NCS were detected as antibody-positive using the Ab ELISA test, which accounted for $6.6 \%$ of all serum samples. Two samples had very similar high values, and the third sample was weakly positive (B2). A doubtful result was obtained for one FBS (B1) sample, which was $2 \%$ of the total number of samples. Of the 45 serum samples, $91.1 \%(n=41)$ were negative, or the level of antibodies was below the limit of detection. Additional designation of tested samples is given in Table 2 .

\section{BVDV antigen detection}

Overall, 3 FBS samples (6.6\%) were positive for BVDV antigen. One of those samples was doubtful (B1), one was weakly positive (B2), and one was negative (F2) for the presence of antibodies in the Ab ELISA.

BVDV antigen was also detected in 18 vaccines. Only one sample among the positive results was not a BVDV vaccine. According to the manufacturer's declaration, it was an inactivated monovalent IBR vaccine.

\section{BVDV genome detection}

RT-PCR

A standard RT-PCR was conducted using four pairs of primers targeting two regions of the BVDV, namely 5'UTR and $\mathrm{N}^{\text {pro }}$. These genome regions are the most used in the molecular characterization of pestiviruses. The BVDV RNA was detected in 26 out of 45 samples $(57.8 \%)$ of sera, and in 5 out of 172 samples $(2.9 \%)$ of collected vaccines. Twelve sera were positive for both regions of BVDV, 5 sera were only positive for the 5'UTR, and 9 samples only for the $\mathrm{N}^{\text {pro }}$ region. Three samples positive in Ag ELISA were also positive in RT-PCR. Detailed results of the standard and real-time RT-PCR for serum are presented in Table 2.

Among vaccine samples, the genetic material of the BVD virus was detected in 4 BVDV vaccines. The fifth vaccine in which the BVDV genome was amplifying was an IBR marker live vaccine, which gave in total $0.58 \%$ of the screened products. The IBR vaccine was not the same vaccine that was positive in the Ag ELISA, and not the same manufacturer.

To check the repeatability of all positive results, we re-extracted serum and vaccine samples and conducted an RT-PCR. The same results were obtained. 


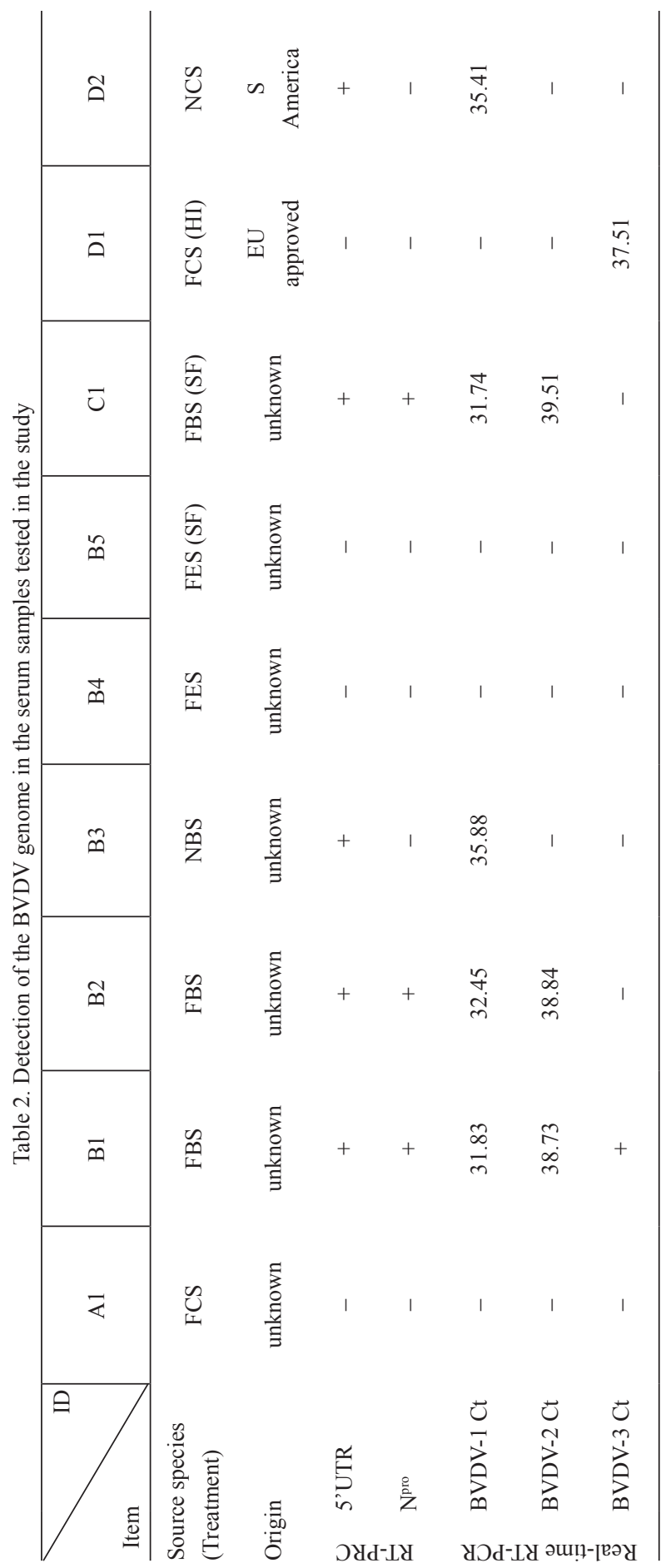




\begin{tabular}{|c|c|c|c|c|c|c|c|}
\hline 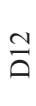 & I & 苞胥 & + & + & 1 & 1 & 1 \\
\hline $\overrightarrow{0}$ & $\widetilde{I}$ & z & 1 & 1 & 1 & 1 & 1 \\
\hline$\stackrel{\circ}{\overrightarrow{0}}$ & $\underline{I}$ & Z & 1 & 1 & 1 & 1 & 1 \\
\hline ดे & $\stackrel{\Omega}{1}$ & z & 1 & 1 & 1 & 1 & 1 \\
\hline$\stackrel{\infty}{\circ}$ & $\begin{array}{l}\text { D̂ } \\
\text { I. }\end{array}$ & 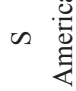 & + & + & 1 & 1 & $\begin{array}{l}\tilde{\sigma} \\
\text { mे }\end{array}$ \\
\hline$\hat{\theta}$ & 足 & \& $\frac{\mathscr{U}}{\mathrm{d}}$ & 1 & 1 & 1 & 1 & లి \\
\hline ๑̊ & 周 & 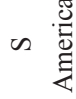 & 1 & + & 1 & 1 & $\begin{array}{l}\text { f } \\
\text { : }\end{array}$ \\
\hline$\hat{n}$ & 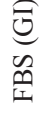 & 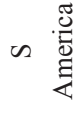 & 1 & 1 & 1 & 1 & $\stackrel{\tilde{m}}{\dot{m}}$ \\
\hline$\stackrel{ \pm}{0}$ & $\stackrel{\Omega}{I}$ & z & 1 & 1 & 1 & 1 & 1 \\
\hline 0 & DI & 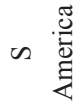 & + & 1 & 1 & 1 & $\begin{array}{l}\infty \\
\dot{m} \\
\dot{m}\end{array}$ \\
\hline
\end{tabular}




\begin{tabular}{|c|c|c|c|c|c|c|c|c|}
\hline$\Xi$ & 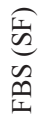 & 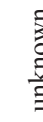 & & + & + & $\dot{m}$ & 1 & 1 \\
\hline I & m & $\sim$ & 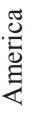 & + & + & $\begin{array}{r}\dot{m} \\
\ddot{m}\end{array}$ & 1 & 1 \\
\hline 厌 & صి & $\sim$ & 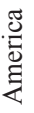 & + & + & ñ & 1 & 1 \\
\hline$\overline{\text { 퍼 }}$ & 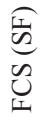 & 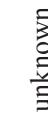 & & 1 & 1 & 1 & 1 & 1 \\
\hline$\frac{\infty}{a}$ & 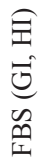 & $\sim$ & 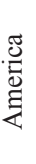 & + & + & 1 & 1 & $\begin{array}{l}\mathcal{O} \\
\dot{+} \\
\text { mे }\end{array}$ \\
\hline$\overline{\bar{\theta}}$ & 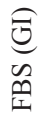 & $\sim$ & 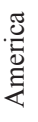 & 1 & 1 & 1 & 1 & $\stackrel{\nabla}{\stackrel{\sim}{m}}$ \\
\hline$\frac{0}{a}$ & D̂ & 吾 & & + & + & $\begin{array}{l}n \\
n \\
\text { ñ }\end{array}$ & 1 & స్ \\
\hline$\frac{n}{a}$ & 公 & 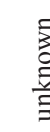 & & + & + & $\frac{\hat{m}}{m}$ & 1 & mे \\
\hline$\frac{\Delta}{\Delta}$ & $\mathscr{I}$ & & 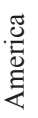 & + & + & $\frac{\mathbb{N}}{\frac{\pi}{m}}$ & 1 & $\stackrel{\Xi}{\Xi}$ \\
\hline$\frac{m}{\vec{\rho}}$ & 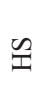 & 范 & $\begin{array}{l}\text { 己્ટ } \\
\frac{\vec{J}}{\mathbb{J}} \\
N\end{array}$ & 1 & 1 & 1 & 1 & 1 \\
\hline
\end{tabular}




\begin{tabular}{|c|c|c|c|c|c|c|c|}
\hline$\stackrel{ }{\exists}$ & D̂ & 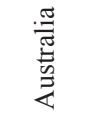 & 1 & + & 1 & 1 & $\begin{array}{l}\stackrel{\infty}{\circ} \\
\dot{m}\end{array}$ \\
\hline શે & 象 & 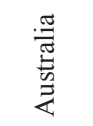 & 1 & + & लें & 1 & $\begin{array}{r}\hat{n} \\
\stackrel{n}{n}\end{array}$ \\
\hline$\stackrel{\infty}{\mathcal{I}}$ & 足 & 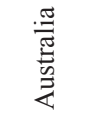 & 1 & + & 1 & 1 & $\begin{array}{l}\infty \\
m \\
m\end{array}$ \\
\hline I & $\frac{n}{1}$ & 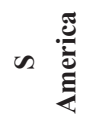 & + & + & 1 & $\stackrel{\infty}{\infty}$ & $\overrightarrow{\dot{m}}$ \\
\hline 우 & Dn & 我 & 1 & + & 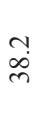 & 1 & $\begin{array}{l}\bar{\sigma} \\
\dot{m}\end{array}$ \\
\hline$\dddot{1}$ & 瓷 & $\infty \frac{\stackrel{\Xi}{E}}{\stackrel{\Xi}{E}}$ & 1 & + & 1 & 1 & $\stackrel{n}{m}$ \\
\hline 声 & 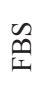 & 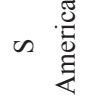 & 1 & + & 1 & 1 & $\begin{array}{l}\stackrel{n}{n} \\
\dot{m}\end{array}$ \\
\hline 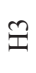 & $\tilde{I}$ & 岕 & 1 & 1 & 1 & 1 & 1 \\
\hline Iี & 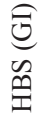 & 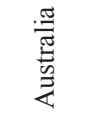 & 1 & 1 & ウ̊. & 1 & ָె \\
\hline $\bar{I}$ & $\stackrel{0}{\oplus}$ & 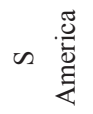 & + & + & $\begin{array}{c}\hat{\infty} \\
\infty \\
m\end{array}$ & 1 & $\begin{array}{l}0 \\
\infty \\
\infty\end{array}$ \\
\hline
\end{tabular}




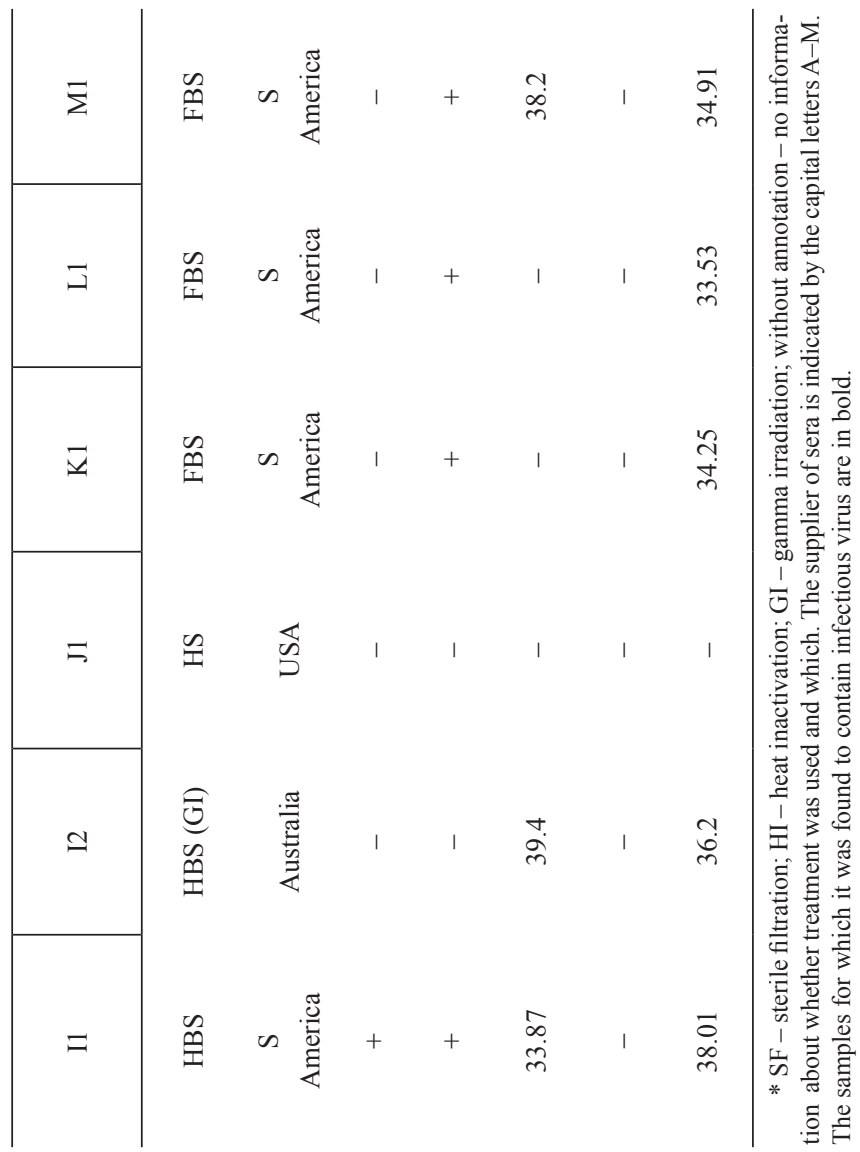




\section{Real-time RT-PCR}

In the real-time RT-PCR test, an additional 8 sera were positive for the presence of the BVDV genome over the 26 detected by the standard RT-PCR test. The results revealed that only one sample, M1 FBS of Australian origin was contaminated with all 3 species of BVD virus. The most frequent virus species in the analyzed sera was BVDV-3 (75.5\%), followed by BVDV-1 (40\%) and BVDV-2 (11.1\%). Of all sera, $44.4 \%(n=20)$ were positive only to a single BVDV species, which was BVDV-1 or BVDV-3. BVDV-2 never occurred alone in the tested sera. In the cases where 2 species of BVDV were detected, most were 1 and $3(n=8)$, fewer were 1 and $2(n=3)$, and the last paired species were 2 and $3(n=1)$.

Positive results were obtained only for 10 out of 17 BVDV vaccines. In these vaccines, the presence of BVDV-1 was confirmed and this was in accordance with the manufacturer's declaration. The contamination with BVDV of the IBR marker live vaccine which was detected by standard was confirmed by real-time RT-PCR, and BVDV species 1 was identified. As in the case of serum and vaccine samples, more positive results were obtained by using real-time RT-PCR than standard RTPCR, which indicates greater usefulness of this method in routine diagnosis.

\section{Infectious status of BVDV in FBS}

After incubation of cell cultures with 33 of BVDV-positive sera in Ag ELISA, standard RT-PCR, and real-time RT-PCR, it could be confirmed that none of the samples contained the cpe biotype of the BVD virus, which was in accordance with the manufacturer's declaration. The frozen cell culture samples were screened for the presence of genetic material of BVDV and only one was positive for it. A positive result in the RT-PCR and real-time RT-PCR $(\mathrm{Ct}=30$ for BVDV-2) indicated the presence of the infectious BVD virus in that particular FBS sample (H7), which had been able to infect the cell culture and replicate. With regard to the information contained in the product specification, the serum batch was standard quality and not gamma irradiated. The obtained RT-PCR product (BVDV isolated in cell culture) was sequenced, analyzed, and compared to the sequence obtained straight from the FBS without propagation in cell culture. The sequence identity for these samples was $90.7 \%$ and both were classified as BVDV-2a. The highest similarity to the sequence from FBS possible to find using the BLAST NCBI tool was demonstrated by the isolate UEL12-Br/17 (GenBank: MG004720) isolated first in southern Brazil in 2017. This also corresponded to the origin of the tested serum, which was claimed to be South America.

\section{Phylogenetic analysis}

The sequences of virus strains used in phylogenetic analyses and their GenBank accession numbers are shown in Figures 1, 2, and 3. Phylogenetic analysis was made to determine the species and subtypes of the isolated BVDV strains. The viral RNA load from only 15 sera and 5 vaccine samples within the 5'UTR region and 12 sera samples within Npro was sufficient to obtain nucleotide sequences for genetic analysis. Phylogenetic analysis of the 5'UTR and $\mathrm{N}^{\text {pro }}$ partial sequences from sera combined with additional sequences from GenBank identified 6 genotypes of BVDV, which were BVDV1a, BVDV-1b, BVDV-1c, BVDV-1d, BVDV-2a, and BVDV-3. Interestingly, there were 
some mismatches between species and subtypes, of BVDV strains regarding the analyzed genome region, namely $5^{\prime} \mathrm{UTR}$ and $\mathrm{N}^{\text {pro }}$. Only in the cases of 2 isolates from sera were the same species and subtypes obtained in both analyzed regions. The explanation for these differences is a mixture of many subtypes of BVDV in one serum batch.

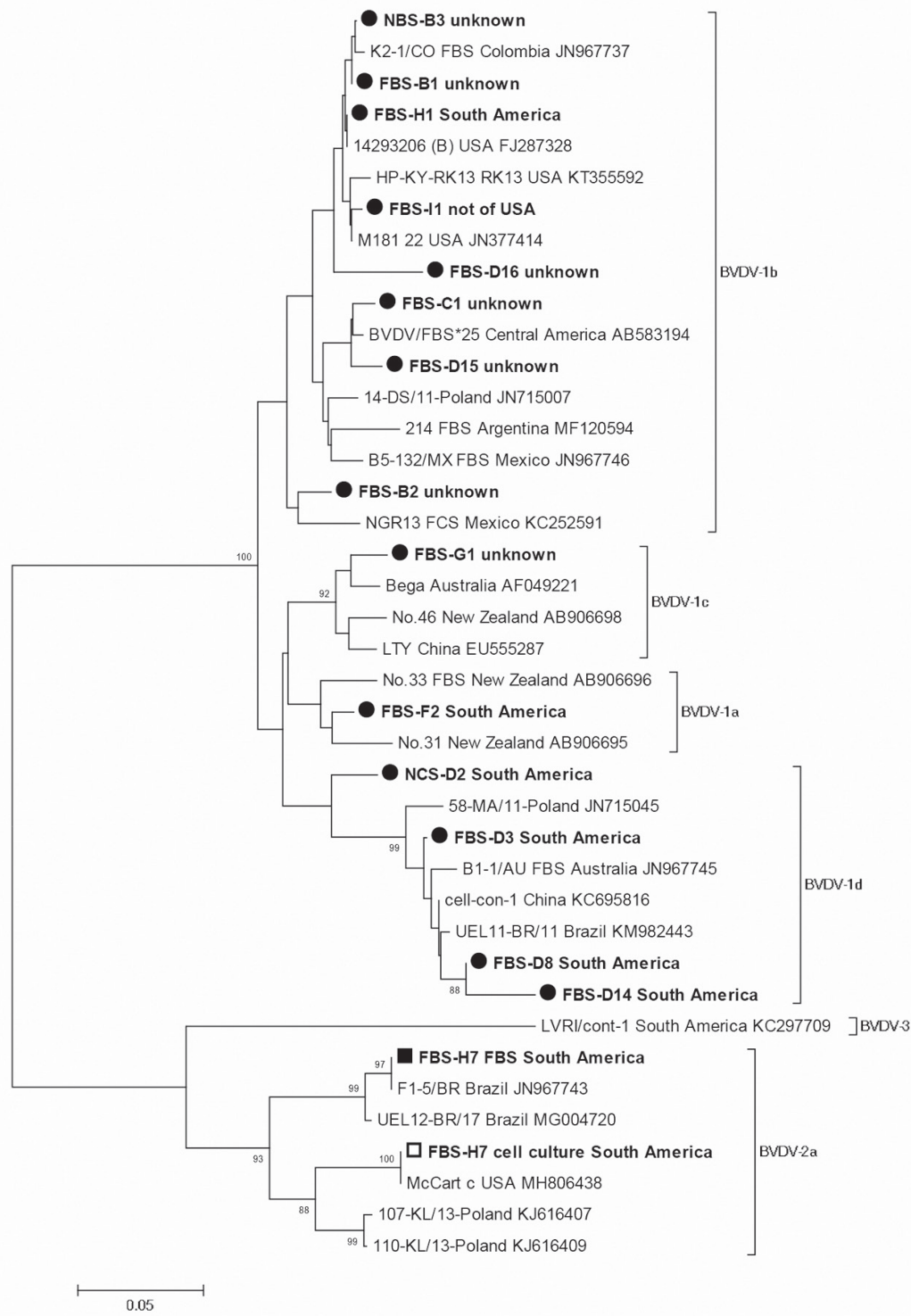

Figure 1. Phylogenetic tree based on the partial 5'UTR sequences obtained from commercial sera. Strains reported in this study are labeled in bold and marked with a black circle, and infectious BVDV strains detected in the FBS sample are denoted by a black square, and this strain isolated in MDBK cell culture carries a white square symbol (GenBank accession numbers: MN513396-MN513411). The numbers close to the major nodes indicate the bootstrap values (in \%). Reference sequences were retrieved from GenBank and country of origin and the accession numbers are listed after each strain 


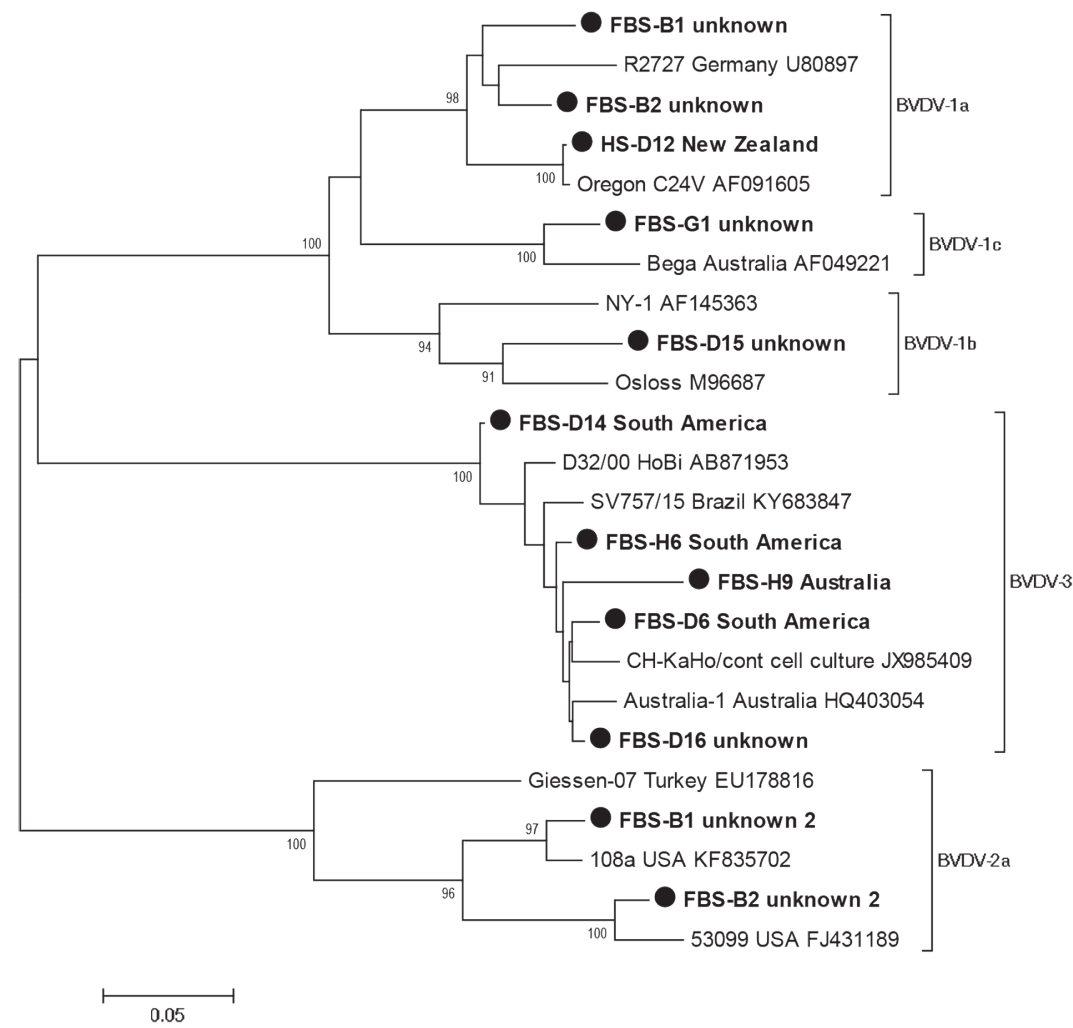

Figure 2. Phylogenetic tree based on the partial $\mathrm{N}^{\text {pro }}$ sequences obtained from commercial sera. Strains reported in this study are labeled in bold and marked with a black circle (GenBank accession numbers: MN513384-MN513395). The presence of the ID of one serum in more than one clade is explained by the presence of several different BVDV species in the tested commercial serum. The numbers close to the major nodes indicate the bootstrap values (in \%). Reference sequences were retrieved from GenBank and country of origin, and the accession numbers are listed after each strain

This is indicated by the presence of many signals present in the background of the main sequence chromatogram. Each genotype showed significant genetic diversity as reflected by the number of nodes and length of branches in trees among the same subtype. The set of panpestivirus primers has a weaker ability to detect atypical pestiviruses (Vilcek et al., 1994), so that is why none could be seen on the tree built on the basis of the 5'UTR region. Thanks to the possibility of using dedicated primer pairs for amplification of the $\mathrm{N}^{\text {pro }}$ region of atypical pestiviruses, a clade was created with five BVDV-3 isolates with clearly visible differences between them. Surprisingly only subtype BVDV-1d was detected, but the comparison of the real-time RT-PCR results demonstrates the content of two different species of BVDV in serum sample D14. From the BVDV-1d clade, sample D14 was the only one for which readable sequences were obtained in both regions, and analysis of the $\mathrm{N}^{\text {pro }}$ region showed that the sequence 
belonged to BVDV-3. Phylogenetic analysis of the nucleotide sequences of the infectious virus isolated from FBS-H7 corresponding to the partial 5'UTR region revealed that the strains (both from FBS and isolated in cell culture) were clustered within the subtype $2 \mathrm{a}$. The $\mathrm{N}^{\text {pro }}$ fragment of the MDBK could not be sequenced.

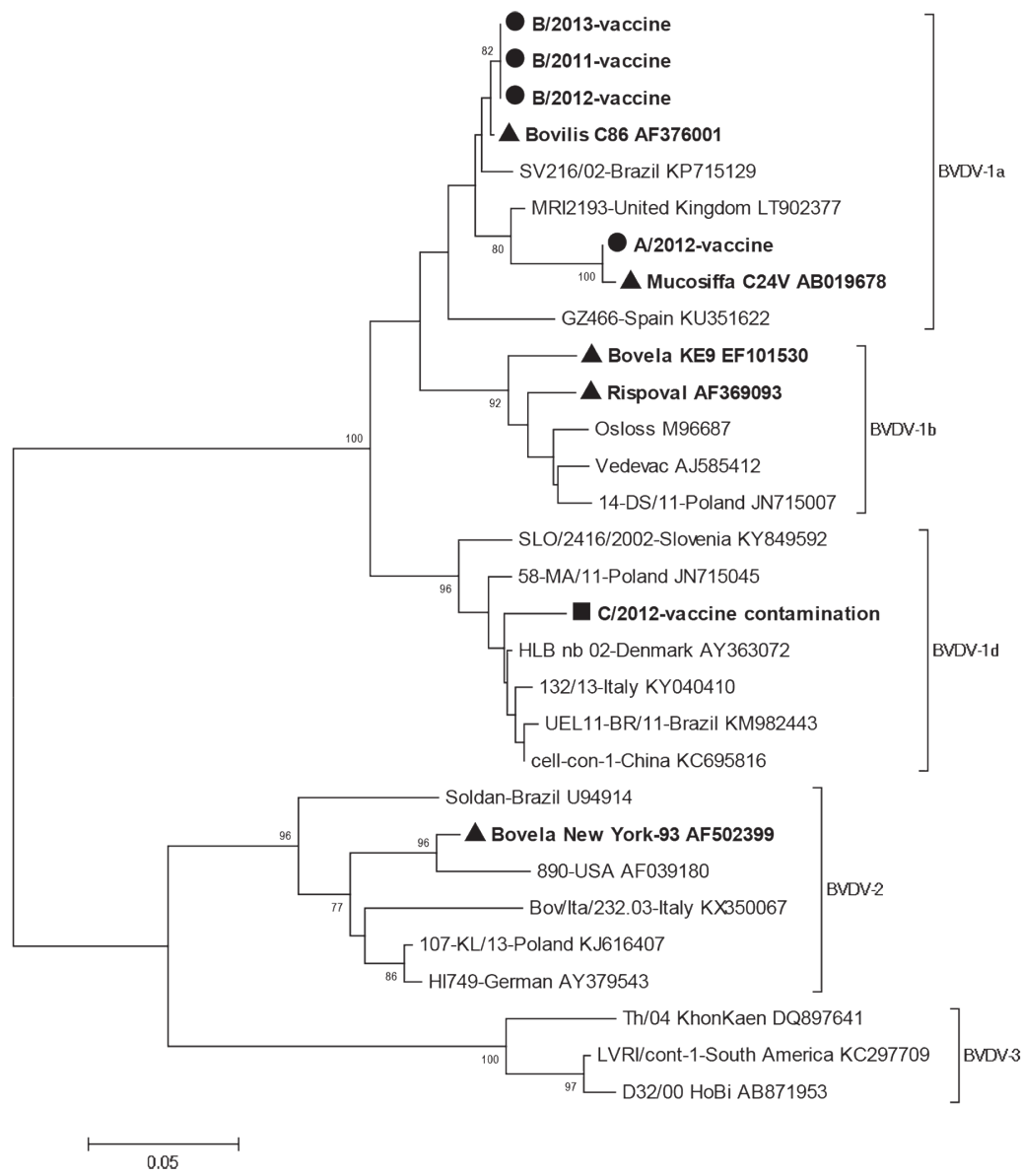

Figure 3. Phylogenetic tree based on partial 5'UTR sequences obtained from tested vaccines (GenBank accession numbers: MN513412-MN513416). Vaccine strains detected in this study are labeled in bold and marked with a black circle. The BVDV found in the IBR vaccine is marked with a black square. The numbers close to the major nodes indicate the bootstrap values (in \%). Reference sequences were retrieved from GenBank and country of origin and the accession numbers are listed after each strain.

Additionally, BVDV vaccine strains are marked with black triangle

Analysis of sequences obtained from vaccines confirms the content of strains that are declared in the leaflet by the producer. The BVDV strain detected in the IBR vaccine number 107 has the highest sequence similarity to isolates from China, Poland, 
Denmark, and, interestingly, from Brazil. Brazilian strain sequence used in phylogenetic analysis, showed the highest sequence identity to BVDV contamination from H7, where an infectious virus was detected.

\section{Discussion}

Viruses used in vaccine production are grown in cell lines, which are supplemented with FBS. Due to the risk of viral contamination associated with the use of bovine serum, it is strongly recommended to inactivate the serum by a validated and efficacious treatment before use, in addition to direct testing for viruses. Most biopharmaceutical companies demand raw FBS is tested for the presence of BVDV before irradiation, which is regulated by the EU EMEA Directive for Pharmaceutical Raw Material of Bovine Origin (EMEA-CPMP-BWP-1793-02). Nevertheless, some companies that want to avoid the risk that their products will not be approved for use in the production of vaccines or other products irradiate raw FBS prior to testing for BVDV, without informing the biopharmaceutical companies. Moreover, they choose tests that are not able to detect inactivated viruses or that are less sensitive (Hawkes, 2016). After comparison of the results from a standard RT-PCR (5'UTR and Npro) with those of a real-time RT-PCR test, we can observe that the panpestivirus primer set was not able to detect all BVDV-3 positive samples, which confirms this finding. Also, it is congruent with the results obtained by others quite recently (Stahl et al., 2010; Peletto et al., 2012) and earlier by Vilcek et al. (1994) who emphasized the problems of intralaboratory contamination and doubtful specificity of primers used in RT-PCR.

It is worthy of note that in this study $73 \%(33 / 45)$ of the commercial sera were positive for at least one BVDV species, based on results in standard RT-PCR and real-time RT-PCR tests. Similarly to what we found for BVDV contamination in sera was previously reported by other research groups, where from $53.4 \%$ to $100 \%$ of tested sera were found contaminated with RNA of at least one pestivirus species (Laassri et al., 2018; Monteiro et al., 2018; Giammarioli et al., 2015), as they also were in our results. In the present work, a very large percentage $(75.5 \%)$ of positive sera were contaminated with BVDV-3, confirming the results obtained in the study by Giammarioli et al. (2015). After comparing the place of origin of those sera we can conclude that they came from the same regions of the world. In contrast, Bauermann et al. (2014), who only studied sera of North American origin, found no evidence for the presence of BVDV-3. We could not confirm this, because of the lack of sera of this origin in our research. Despite the fact that the majority of positive sera were contaminated with BVDV-3, most of BVDV's in Poland are representatives of species 1. According to studies from 2013 (Kuta et al., 2013 b), the majority of isolates were subtypes $1 \mathrm{~b}$ and $1 \mathrm{~d}$, while subsequent studies (Mirosław and Polak, 2019) showed the majority of $1 \mathrm{~b}, 1 \mathrm{~g}, 1 \mathrm{f}$, and $1 \mathrm{~d}$. While no BVDV-3 infected animals have been detected in Poland, the presence of BVDV-3 in the country cannot be ruled out. Clinical signs are indistinguishable from infections with typical field strains and 
not all diagnostic tests are able to discriminate between typical BVDV and BVDV-3 infections. Moreover, not every sample from a BVDV positive animal is classified for further analysis, which is often associated with a deficiency of sufficient material. Though local vaccines contain BVDV-1a, 1b, and BVDV-2, it is unlikely that the virus species occurring in Poland are associated with vaccine virus reversion.

The results obtained by our research group and other researchers emphasize how widely this virus is spread among the animal population in the world. It also indicates the scale of the problem in attempts to eradicate the virus from cattle stock, especially given that biosecurity principles are not always respected, which significantly hampers the success of a program. Reintroduction of atypical pestivirus into BVDV-free herds or where control programs are being introduced would result in massive detriment to animal welfare and economic losses. Contaminated FBS used for quality control and surveillance assays, as well as other research and industrial applications can alter or destroy scientific results or products. Depending on the virus strain and host factors, the clinical manifestation of infection can vary from asymptomatic (the most common) or mild to severe. The consequences of infection may include reproduction failure, poor reproductive performance, increased susceptibility to secondary infections due to the immunosuppressive effect of BVDV, congenital abnormalities, and the birth of PI calves which serve as virus reservoirs.

Introduction of HoBi-like viruses could occur via live vaccines (Falcone et al., 2003) or insufficiently inactivated killed vaccines (Stahl et al., 2010). Previously, BVDV-2 contaminating the BoHV-1 marker vaccine was shown to cause widespread outbreak of BVD in the Netherlands and Italy (Barkema et al., 2001). In this study, BVDV antigens were found in inactivated monovalent IBR vaccine, and BVDV-1d partial genome was in an IBR marker live vaccine, which further confirms previous reports and highlights the need for monitoring of bioproducts at each stage of production to prevent a possible outbreak of disease. However, in this particular case, the infectious status of both vaccines could not be checked, because it transpired that the adjuvants used in them were toxic to cell culture. Nevertheless the risk of disease or seroconversion after using such vaccines is high.

Despite the fact that in our studies over $70 \%$ of tested sera proved to be contaminated by BVDV, only one serum showed the presence of an infectious virus. It is possible that the presence of the detected specific antibodies against BVDV in high titers in the cases of 3 sera may have affected the result of virus isolation by neutralization, and resulted in false negative results. The virus was ncp, like most field isolates (Deregt and Loewen, 1995). Two nucleotide sequences were obtained from this serum, one directly from FBS by sequencing of the RT-PCR product, and the second after replication of the virus in cell culture for 3 passages. The large difference of sequence identity of $9.3 \%$ between these two sequences may be due to the fact that the given FBS sample was a pool of sera taken from many animals that may have been exposed to different isolates of the same BVDV species. For this reason, it could contain several virus isolates, as evidenced by the presence of additional peaks in the background in the obtained sequencing chromatogram (direct from FBS). Nevertheless, we obtained a clear sequencing chromatogram with no interference in the background for virus isolated in cell culture. This proves that FBS contains 
only one isolate of infectious BVDV. The serum in which the BVD infectious virus was detected was claimed to be of EU-approved origin. No connection was observed between the place of origin or purification method used and the occurrence of contamination, which was also noticed by Hawkes (2015). Many commercial sera were cleaned by filtration, but due to the small particle size of BVDV (45-55 nm), many viral particles could remain in commercial FBS after standard sterile filtration. It means that we should not use animal-derived products for diagnostic, medical or production purposes without using additional safety measures such as, for example, additional heat inactivation or gamma irradiation.

It seems that the perfect solution for avoiding pathogen contamination is using serum-free media. They were developed to cut down the use of animals in research and the medical industry. These products have met with great success, especially in the production of some proteins for medical use. Nevertheless, pharmaceutical companies, diagnostic centers, and researchers still depend on animal-derived products like FBS for most of their cell culture needs, because we are not yet able to replicate all growth factors present in FBS or to produce serum-free media on a large scale (Hawkes, 2015).

The current study provides evidence that the contamination of bioproducts with all species of BVDV, and especially the contamination of commercial FBS available on the local market and used in immunoprophylaxis and diagnostics in Poland, remains a threat for pestivirus transmission and may falsify the results of a diagnostic test based on commercial serum usage. While the risk of pestivirus infection through a vaccine seems low, the use of the vaccines contaminated with the BVDV antigen may provoke seroconversion, which may result in failure of a BVDV eradication program.

\section{References}

A u d e t S.A., C ri m R.L., B e e l e r J. (2000). Evaluation of vaccines, interferons and cell substrates for pestivirus contamination. Biologicals, 28: 41-46.

B arkema H.W., B artels C.J., van Wuijckhuise L., Hesselink J.W., Holzhauer M., Weber M.F., Franken P., Kock P.A., Brus ch k e C.J., Zi m m e r G.M. (2001). Outbreak of bovine virus diarrhea on Dutch dairy farms induced by a bovine herpesvirus 1 marker vaccine contaminated with bovine virus diarrhea virus type 2. Tijdschr. Diergeneeskd., 126: 158-165.

B a u ermann F.V., F lores E.F., Rid p ath J.F. (2012). Antigenic relationships between Bovine viral diarrhea virus 1 and 2 and HoBi virus: possible impacts on diagnosis and control. J. Vet. Diagn. Invest., 24: 253-261.

B a u ermann F.V., F 1 or es E.F., F a l k e n berg S.M., We ible n R., R id path J.F. (2014). Lack of evidence for the presence of emerging HoBi-like viruses in North American fetal bovine serum lots. J. Vet. Diagn. Invest., 26: 10-17.

Baxi M., McRae D., Baxi S., Greiser-Wilke I., Vilcek S., A moako K., Deregt D. (2006). A one-step multiplex real-time RT-PCR for detection and typing of bovine viral diarrhea viruses. Vet. Microbiol., 116: 37-44.

Cortez A., He inemann M.B., de Castro A.M.M.G., Soares R.M., Pinto A.M.V., A 1fi eri A.A., F lores E.F., Le i te R.C., R i chtzen ha in L.J. (2006). Genetic characterization of Brazilian bovine viral diarrhea virus isolates by partial nucleotide sequencing of the 5'-UTR region. Pesq. Vet. Bras., 26: 211-216. 
Decaro N., Lucente M.S., Mari V., Cirone F., Cordioli P., Camero M., Sciarrett a R., L o surdo M., L or us s o E., B u on ava gli a C. (2011). Atypical pestivirus and severe respiratory disease in calves, Europe. Emerg. Infect. Dis., 17: 1549-1552.

D e re g t D., L o ew en K.G. (1995). Bovine viral diarrhea virus: biotypes and disease. Can. Vet. J., 36: $371-378$.

Falcone E., Cordioli P., Tarantino M., Muscillo M., Sala G., La Rosa G., Archetti I.L., Marianelli C., L o mbardi G., Tollis M. (2003). Experimental infection of calves with bovine viral diarrhoea virus type-2 (BVDV-2) isolated from a contaminated vaccine. Vet. Res. Commun., 27: 577-589.

Giammarioli M., Ridpath J.F., Ross i E., Bazzucchi M., Cas ciari C., De Mia G.M. (2015). Genetic detection and characterization of emerging HoBi-like viruses in archival foetal bovine serum batches. Biologicals, 43: 220-224.

Gi a n g s pero M., Har a s a w a R., Verhuls t A. (1997). Genotypic analysis of the 5'-untranslated region of a pestivirus strain isolated from human leukocytes. Microbiol. Immunol., 41: 829-834.

Giangaspero M., Vacirca G., Harasawa R., Büttner M., Panuccio A., De Giuli Morghen C., Zanetti A., B elloli A., Verhulst A. (2001). Genotypes of pestivirus RNA detected in live virus vaccines for human use. J. Vet. Med. Sci., 63: 723-733.

H a w k s P.W. (2015). Fetal bovine serum: geographic origin and regulatory relevance of viral contamination. Bioresour. Bioprocess, 2: 34.

H a w k e s P.W. (2016). Fetal Bovine Serum (FBS). Irradiation Working Group. Paper was written with input from: GE Life Sciences (HyClone Laboratories), SAFC (Sigma Aldrich), and Biowest, https:// www.biowest.net/media/biowest_topics_for_discussion_analysis_chapter_8_066350600_ 0934_20052016.pdf, Access date: $\overline{20}$ May $\overline{2} 01 \overline{6}$.

IDEXX Laboratories. (2005). Bovine Viral Diarrhea Virus Antigen Serum/Plus Test Kit Validation Data Report. Westbrook, Maine. On file at IDEXX Laboratories, Inc.

K u ta A., P olak M.P., L a r s k a M., Ż m u d z iń s k i J.F. (2013 a). Monitoring of Bovine Viral Diarrhoea Virus (BVDV) infection in Polish dairy herds using bulk tank milk samples. Bull. Vet. Inst. Pulawy, 57: 149-156.

K u ta A., P o la k M.P., L a r s k a M., Ż m u dzińs ki J.F. (2013 b). Predominance of bovine viral diarrhea virus $1 \mathrm{~b}$ and $1 \mathrm{~d}$ subtypes during eight years of survey in Poland. Vet. Microb., 166: 639-644.

La as sri M., Mee E.T., Connaughton S.M., Manukyan H., Gruber M., Rodriguez- Hernandez C., Minor P.D., S chepe $1 \mathrm{~m}$ ann S., Chumakov K., Wood D.J. (2018). Detection of bovine viral diarrhoea virus nucleic acid, but not infectious virus, in bovine serum used for human vaccine manufacture. Biologicals, 55: 63-70.

L a $n$ y o n S.R., A n d e r s o n M.L., B e r g m a n E., R e i c h e 1 M.P. (2013). Validation and evaluation of a commercially available ELISA for the detection of antibodies specific to bovine viral diarrhoea virus (bovine pestivirus). Aust. Vet. J., 91: 52-56.

L i u L., X i a H., B e lá k S., B a u l e C. (2008). A TaqMan real-time RT-PCR assay for selective detection of atypical bovine pestiviruses in clinical samples and biological products. J. Virol. Methods, 154: $82-85$.

L i u L., X i a H., B a u l e C., B e l a k S. (2009). Maximum likelihood and Bayesian analyses of a combined nucleotide sequence dataset for genetic characterization of a novel pestivirus, SVA/cont- 08 . Arch. Virol., 154: 1111-1116.

Mirosław P., P o la k M. (2019). Increased genetic variation of bovine viral diarrhea virus in dairy cattle in Poland. BMC Vet. Res., 15: 278.

Monteiro F.L., Cargnelutti J.F., Braunig P., Folgueras-Flatschart A.V., Santo s N.C., P it u c o E.M., We iblen R., Flore s E.F. (2018). Detection and genetic identification of pestiviruses in Brazilian lots of fetal bovine serum collected from 2006 to 2014. Pesq. Vet. Bras., 38: 387-392.

Nogue ir a F.C.S., Vel a s quez E., M e lo A.S.O., D o mont G.B. (2016). Zika virus may not be alone: proteomics associates a bovine-like viral diarrhea virus to microcephaly. Biorxiv, doi: http:// dx.doi.org/10.1101/062596.

Pecora A., Perez Aquirreburualde M.S., Ridpath J.F., Dus Santos M.J. (2019). Molecular characterization of pestiviruses in fetal bovine sera originating from Argentina: evidence of circulation of HoBi-like viruses. Front. Vet. Sci., 6: 359. 
Peletto S., Zuccon F., Pitti M., Gobbi E., Marco L.D., Caramelli M., Ma soero L., A c u t is P.L. (2012). Detection and phylognetic analysis of an atypical pestivirus, strain IZSPLV To. Res. Vet. Sci., 92: 147-150.

P o la k M.P., R o la J., Ż m u d z i ńs k i J.F. (2008). Contamination of foetal bovine serum with bovine viral diarrhoea virus (BVDV). Bull. Vet. Inst. Pulawy, 52: 501-505.

Polak M.P., Kuta A., Rybałtowski W., Rola J., Larska M., Żmudziński J.F. (2014). First report of bovine viral diarrhoea virus-2 infection in cattle in Poland. Vet. J., 202: 643-645.

Rypuła K., Kumala A., Kaba J., Płoneczko-Janeczko K., Wojewoda-Kotwic a B., M a z urki ew i c z M. (2010). Epidemiological aspects of BVDV infections in dairy cattle herds in Poland. Med. Weter., 66: 684-687.

S a s a k i T., Haras aw a R., Sh intani M., Fujiw ara H., S a s a ki Y., Horino A., Kenri T., A s a d a K., K a to I., Ch in o F. (1996). Application of PCR for detection of mycoplasma DNA and pestivirus RNA in human live viral vaccines. Biologicals, 24: 371-375.

S c h ir r m e i e r H., S tre b e l ow G., D e pner K., H offm a n n B., B e e r M. (2004). Genetic and antigenic characterization of an atypical pestivirus isolate, a putative member of a novel pestivirus species. J. Gen. Virol., 85: 1647-3652.

S mith D.B., M e yers G., Bukh J., Gould E.A., Mon ath T., Muerhoff A.S., P let nev A., R i c o-Hes s e R., S t a plet on J.T., S i m monds P., B e c her P. (2017). Proposed revision to the taxonomy to the genus Pestivirus, family Flaviviridae. J. Gen. Virol., 98: 2106-2112.

Stahl K., Kampa J., Alenius S., Persson Wasman A., Baule C., Aiumlamai S., B e la k S. (2007). Natural infection of cattle with an atypical 'HoBi'-like pestivirus - implications for BVD control and for the safety of biological products. Vet. Res., 38: 517-523.

St a hl K., B e er M., S chirrmeier H., Hoffm an n B., B el a k S., A l e ni us S. (2010). Atypical 'HoBi'-like pestiviruses - recent findings and implications thereof. Vet. Microbiol., 142: 90-93.

Studer E., B ertoni G., C a ndrian U. (2002). Detection and characterization of pestivirus contaminations in human live viral vaccines. Biologicals, 30: 289-296.

Top lak I., S a ndvik T., B a r li c-Magan ja D., Grom J., P a to n D.J. (2004). Genetic typing of bovine viral diarrhoea virus: most Slovenian isolates are of genotypes $1 \mathrm{~d}$ and $1 \mathrm{f}$. Vet. Microbiol., 99: 175-185.

Van Ma an en C. (2001). Deventer validation report - Bovine Viral Diarrhea Virus (BVDV) antigen detecting ELISAs. Deventer, The Netherlands: Animal Health Service.

Vil c e k S., H e r r ing A.J., H e r r ing J.A., N e t t l e t o n P.F., L o w in g s J.P., P a t o n D.J. (1994). Pestivirus isolated from pigs cattle and sheep can be allocated into at least three genogroups using polymerase chain reaction and restriction endonuclease analysis. Arch. Virol., 136: 309-323.

Vilcek S., Durkovic B., Koles arova M., P aton D.J. (2005). Genetic diversity of BVDV: Consequences for classification and molecular epidemiology. Prev. Vet. Med., 72: 31-35.

Weber M.N., Silveira S., Machado G., Groff F.H.S., Mos ena A.C.S., Budas zew s ki R.F., D up on t P.M., C orbellin i L.G., C a n a 1 C.W. (2014). High frequency of bovine viral diarrhea virus type 2 in Southern Brazil. Virus Res., 191: 117-124.

Wernicki A., Urban-Chmiel R., S tęgierska D., Adaszek Ł., Kalinowski M., Puc hals k i A., D e c M. (2015). Detection of the bovine viral diarrhoea virus (BVDV) in young beef cattle in eastern and south-eastern regions of Poland. Pol. J. Vet., 18: 141-146.

Wernike K., Gethmann J., Schirrmeier H., Schröder R., Conraths F.J., Beer M. (2017). Six years (2011-2016) of mandatory nationwide bovine viral diarrhea control in Germany - a success story. Pathogens, 6: 50.

W o $1 \mathrm{f}$ G. (2007). The perfect BVDV diagnosis? Proc. Symposium on Diagnosis of BVDV in Ear Notch Samples. Stendal, Germany.

Yolken R., Dubovi E., Le is ter F., Reid R., A l meido-Hill J., S a n to sham M. (1989). Infantile gastroenteritis associated with excretion of pestivirus antigens. Lancet, 1: 517-520.

Received: 24 X 2019

Accepted: 18 V 2020 